\title{
APROXIMACIÓN DESDE LOS ESTILOS COGNITIVOS A LA SOLUCIÓN DE PROBLEMAS EN DISEÑO' ${ }^{1}$
}

\author{
Fernando Álvarez ${ }^{2}$ \\ Magíster en Pedagogía \\ Profesor Asociado del programa \\ de Diseño Industrial \\ Universidad Jorge Tadeo Lozano \\ fernando.alvarez@utadeo.edu.co
}

\author{
Edgar Martínez ${ }^{3}$ \\ Magíster en Pedagogía de la tecnología \\ Profesor del Magíster del programa de Diseño \\ Industrial \\ Universidad Jorge Tadeo Lozano \\ edgar.martinez@utadeo.edu.co
}

\section{Resumen:}

La investigación elaboró un panorama conceptual para establecer elementos de la formulación y solución creativa de problemas como capacidades cognitivas orientadas hacia una reflexión pedagógica y didáctica para la disciplina del diseño industrial. Se consideraron los aportes de la psicología diferencial, la cual ha caracterizado los estilos cognitivos y se constituye en una mirada pertinente para entender la "heterogeneidad estudiantil". Esta perspectiva que cuenta con resultados en distintos continentes puede ofrecer otro punto de vista acerca de cómo procurar la inclusión académica entendiendo la diversidad y el entendimiento de factores relacionados con la deserción estudiantil.

El estudio realizó una caracterización inicial acerca de las dimensiones de los estilos sensibilidad-independencia a los estudiantes de diseño industrial y, posteriormente, se centró en la caracterización de los estilos desde la solución creativa de problemas como capacidad cognitiva implicados en el desarrollo de proyectos.

Palabras Clave: estilos cognitivos, diseño industrial, solución creativa de problemas, pedagogía.

\begin{abstract}
:
the research made a conceptual panorama to establish elements of the creative formulation and solution of problems as cognitive capacities oriented to a pedagogical and didactical reflection to the discipline of industrial design where were considered the contributions of the perspective of differential psychology which has characterized the cognitive styles and is a pertinent view to understand the student heterogeneity.

The study made an initial characterization about the dimensions of the sensibility-independence styles to the students of industrial design and then was focused in the characterization of the styles from the creative solution of problems as cognitive capacity implied in the project development.
\end{abstract}

Keywords: cognitive styles, Industrial Design, subjectivity, design processes, educability. 


\section{Introducción}

Este trabajo hace parte de una de las cinco etapas de realización de un proyecto más amplio realizado en el Programa de Diseño Industrial de la Universidad Jorge Tadeo Lozano (UJTL). Intentó establecer las competencias requeridas para la innovación caracterizando aspectos de la capacidad de diseño, específicamente en el desarrollo de proyectos de diseño industrial, centrado en la línea del diseño basado en tecnología.

La primera etapa finalizada abordó la competencia de las modalidades de procesamiento de la información en las dimensiones de Independencia y Sensibilidad ${ }^{4}$ al campo (SIC) (Álvarez R. \& Martínez S., 2009a,b; 2010a,b,c) dentro de la teoría de los estilos cognitivos (EC) ampliamente trabajada por Hederich y colaboradores en Colombia (Hederich Martínez C., Camargo Uribe, Guzmán Rodriguez, \& Pacheco Giraldo, 1993 a 2008).

A continuación se presentan los resultados obtenidos en la aplicación del test SG-EFT (test de las figuras enmascaradas), el cual ha sido el principal instrumento empleado para la medición mundial de las polaridades (SIC). La prueba ha sido ampliamente utilizada en el territorio colombiano ${ }^{5}$ en la versión de investigación de Hederichet al. (1995).

Este instrumento tiene una confiabilidad de alfa de Cronbach $(0.91 \text { a } 0.96)^{6}$ y un valor corregido Spearman - Brown de $(0.9412)^{7}$. La Tabla 1 presenta cada uno de los estadísticos descriptivos empleados en la caracterización de los resultados obtenidos para un número de 125 pruebas validas.

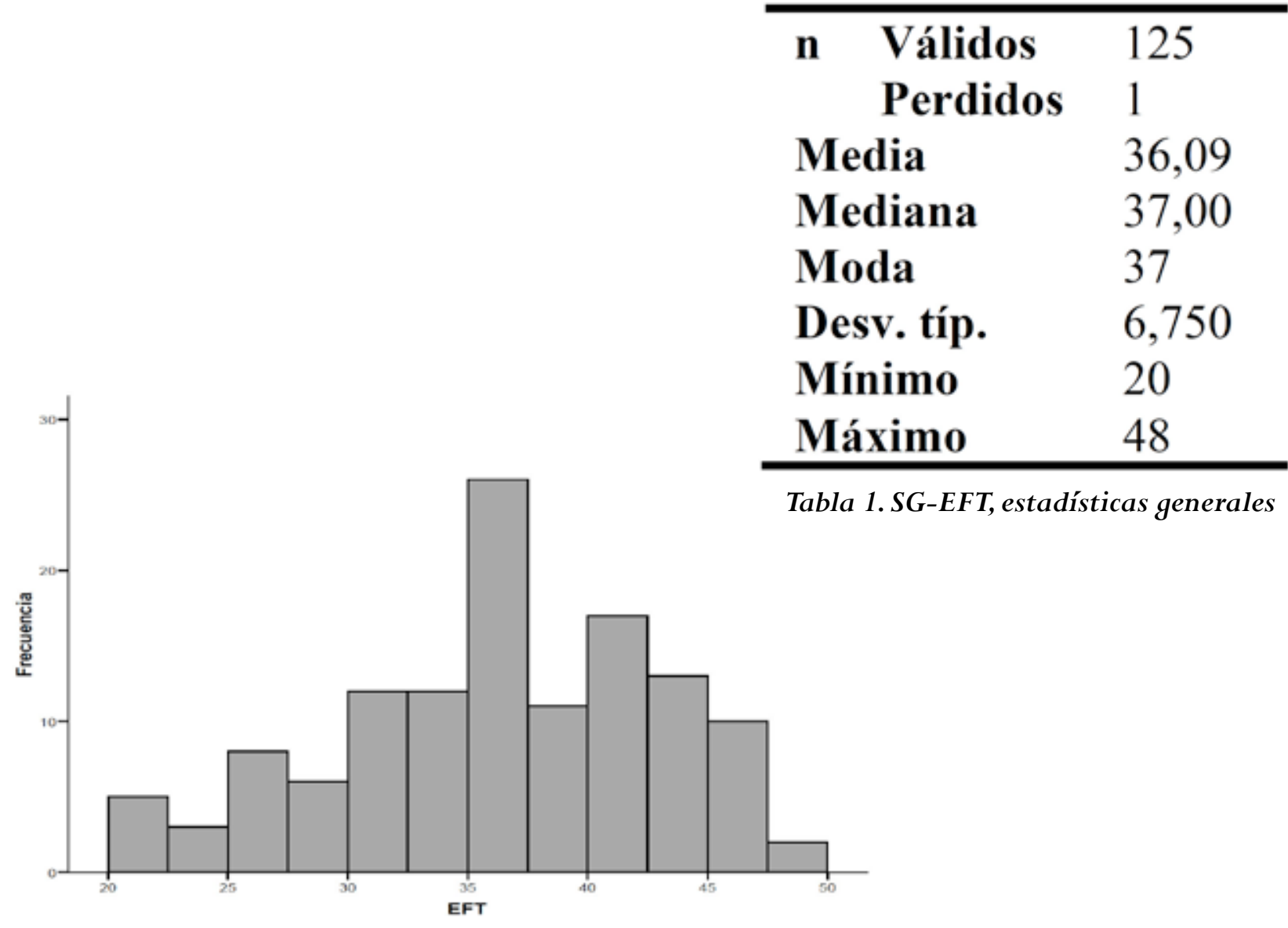

Figura 1. Distribución de frecuencias del SG-EFT. 
Los resultados indicaron de manera consistente que la muestra de estudiantes presenta una tendencia hacia la independencia al campo, ya que en la prueba SG-EFT, se considera que puntaje tendiente a los 50 puntos es un estilo altamente independiente y por el contrario un puntaje cercano a cero se considera un estilo tendiente a la sensibilidad al campo.

El promedio obtenido en la prueba de 36,09 indica una tendencia de los estudiantes de diseño Industrial de la UJTL, lo cual pone de manifiesto la necesidad de considerar la equidad en la enseñanza para los dos estilos; mejor aún, considerar que una parte de la población de estudiantes tiene un estilo que puede no estar siendo considerado en el programa académico ni en las didácticas para la enseñanza. En todo caso se sugiere profundizar aun más el campo de los estilos y su influencia en el ámbito del diseño, ya que no se observó un equilibrio en las polaridades SIC.

\section{El contexto de la solución creativa de problemas}

Bajo el anterior resultado presentado brevemente, se realizó un nuevo estudio en el que se hizo una revisión documental de la investigación acerca de la capacidad para la formulación y solución de problemas dentro del desarrollo de proyectos para diseño. Esto resulta interesante para su aproximación a las competencias del Diseñador Industrial. Conjuntamente con otras características se podría apreciar cuáles favorecen el pensar y actuar creativo.

Conocer la estructura que lleva a la formulación y solución de problemasdesde un enfoque cognitivo ha permitido encontrar algunos estudios contemporáneos y hallazgos importantes relacionados con el campo de la solución creativa de problemas (CPS) ${ }^{8}$, el desarrollo tecnológico, la capacidad de diseño, entre otros.

En el contexto contemporáneo es posible apreciar algunas inconsistencias en las estrategias pedagógicas, didácticas y evaluativas, las cuales vienen afectando la delimitación, desarrollo y concreción en procesos de aprendizaje de la disciplina proyectual (Mazzeo, C., \& Romano, A. M.; 2007). Esto se evidencia en los ambientes educativos desde el estilo de enseñanzaparticular del docente (Sternberg, R., \& Zhang, L.-f.; 2001), tendiendo a la redundancia de isomorfismos y algoritmos en las estrategias de enseñanza. Allí donde el profesor es la única fuente de conocimiento 9 .

En contraste, se tienen los estilos de aprendizaje (Sternberg, R., \& Zhang, L.-f.; 2001), en los cuales los estudiantes, con un estilo cognitivo afín al del profesor, estarían favorecidos en procesos de aprendizaje reflejados en su logro académico; en tanto que los estudiantes con diferente estilo al del profesor tendrían algunas dificultades y bajos logros sobre sus compañeros(Hederich C., Camargo, Guzmán, \& Pacheco, 1995).

Es necesario destacar que el contexto de esta experiencia investigativa surge en el Proyecto Educativo de la Universidad Jorge Tadeo Lozano y el Proyecto educativo del Programa de Diseño Industrialque acoge una gran variedad de perfiles estudiantiles, ya que en su propósito formativo se apuesta por una educación incluyente, para todo el que desee aprender. Esta apuesta sin duda, requiere entender las variadas posibilidades que se han investigado para formular y solucionar problemas con el fin de enseñar en consecuencia dentro del plan de formación de diseñadores industriales.

\section{Problema de investigación}

La aproximación inicial es la comprensión de las características del proceso de construcción que va de la formulación del espacio de problema hacia la síntesis incremental de las alternativas de solución. Inicialmente, se enmarca en un escenario de "problema débilmente estructurado", proceso que pasa por la construcción de la estructura del problema. Estos procesos mentales sobre la solución del problema tienen imbricadas la prefiguración de la solución a partir del uso de signos y el planteamiento de rutas de navegación y exploración de heurísticas, que luego implican la configuración de lo concreto como alternativas de solución(Bachelard, 1994; Andrade \& Lotero, 1998; Boden, 1994; Goel \& Pirolli, 1992).

La estructuración de un problema atraviesa un conjunto de actividades físicas, emocionales y principalmente mentales que lo determinan. La percepción, identificación y formulación de un problema por ejemplo, influyen en la posibilidad de determinar aspectos del problema(Maldonado \& Quintero, 2006). De igual forma, pasar de lo abstracto 
a la posibilidad de una concreción o solución posible implica una correspondencia entre las alternativas de diseño y la formulación de un problema (Bachelard, 1994; Schraagen, 1993).

En este sentido, cuando se estructura de modo complejo el sistema de la situación de diseño es evidente una interiorización en la mente del individuo, del proyecto y a partir de ahí es que e puede especializar y evaluar con argumentos los aspectos de éste (Vigotsky, 1995; García, 2006). Por tanto, existen algunos elementos a considerar en la arquitectura de un problema que pueden influir en las respuestas de diseño. Por tal motivo, las soluciones en la capacidad de diseño pueden presentarse como isoformismos, no como condiciones arquetípicas de la solución, sino como modificaciones superficiales, novedades debido a orientaciones cognitivas para delimitar y dominar las determinantes y requerimientos fundamentales para una solución de diseño (Ivañez, 2000, pág. 81; Krippendorff, 2006).

Con lo presentado anteriormente, una pregunta que recoge este proceso complejo sería: ¿Cómo estos procesos se convierten en competencias clave para el diseño centrado en la innovación tecnológica?

\section{Objetivos y metodología del estudio}

El objetivo general del estudio consistió en realizar una caracterización de la competencia de formulación y solución de problemas centrada en la estructuración del espacio de problema, como capacidad cognitiva en el desarrollo de proyectos de diseño industrial. Fue basado en tecnología como parte del estudio de competencias para la innovación. Los aspectos metodológicos se describen a continuación:

\section{Metodología}

Para el desarrollo de la propuesta se emplearon técnicas de análisis bibliométrico y de análisis de contenido, mediante teorías sobre la cognición, la creatividad y el diseño a través de una aproximación a las bases de datos.

Parte del desarrollo del proyecto implicó la revisión documental de algunos autores especialistas en pedagogía de la tecnología como Andrade \& Lotero, 1998; Goel \& Pirolli, 1992; Layton, 1993 que permitieron fundamentar nuestro abordaje.

Vale la pena señalar que se evidenció en la revisión la presencia de un número significativo de disciplinas comprometidas con la innovación, entre las cuales se destacan los centros de psicología, las universidades y variados sectores industriales interesados en la comprensión del funcionamiento de los procesos involucrados en la CPS, el diseño y la tecnología. Esto con el fin de desarrollar metodologías y productos de diseño para responder a mercados cada vez más competitivos. 


\section{La solución de problemas en la investigación reciente}

Para realizar la búsqueda de referencias sobre trabajos similares se establecieron las palabras clave relacionadas con la temática y se procedió a revisar bases de datos multidisciplinarias como ISI web of knowledge, en Estados Unidos y Europa, REDALyC para Latinoamérica y el Caribe; por último, Scielo lo mismo que Dialnet en un ámbito iberoamericano. Así mismo, se revisaron obras de autores con amplia trayectoria en educación, cognición y diseño ${ }^{10}$.

Las lecturas empleadas en este trabajo bibliométrico fueron seleccionadas conforme al planteamiento del problema de investigación y sus objetivos. Las búsquedas fueron realizadas en español e inglés. A continuación se presenta una serie de tablas que dan cuenta del trabajo de revisión.

\section{a. Palabras Clave}

Los términos de búsqueda empleados se presentan en la Tabla 1 y consisten en una serie de conceptos que parten de enunciar el proceso de formulación hasta el proceso de solución de incremental de problemas; también se especificó el campo disciplinar y epistémico (diseño y tecnología) específico del ámbito de indagación.

Tabla 2. Entradas clave empleadas en la búsqueda en bases de datos.

\begin{tabular}{l}
\hline Palabras clave \\
\hline Formulación de problemas en tecnología y diseño, enfoque cognitivo \\
Solución de problemas en diseño \\
Problemas débilmente estructurados \\
Formulación del espacio de problema de diseño \\
Síntesis incremental en la solución de problemas de diseño \\
\hline
\end{tabular}

En la Tabla 3 se presentan los resultados por los términos de las búsquedas que incluyen las combinatorias de los conceptos de la Tabla 2. Con ello se observa un total de 16 resultados. Se puede destacar que a pesar de las múltiples variaciones entre los términos, la producción especializada al respecto no es tan abundante como podría pensarse. Bajo el término de formulación de problemas para diseño, desde un enfoque cognitivo, se recopilaron cuatro artículos, siendo el mayor número en esta búsqueda en los artículos en español.

Tabla 3. Resultados obtenidos por palabras clave en español.

\begin{tabular}{ll}
\hline Palabras clave con resultados & 2 \\
\hline Formulación de problemas en diseño & 4 \\
Formulación de problemas para diseño, enfoque cognitivo & 2 \\
Solución de Problemas en diseño, enfoque cognitivo & 3 \\
Solución de problemas para diseño & 2 \\
Formulación de problemas para diseño industrial, enfoque cognitivo & 1 \\
Formulación de problemas en tecnología, enfoque cognitivo & 1 \\
Solución de problemas en tecnología, enfoque cognitivo & 1 \\
Síntesis incremental en la solución de problemas de diseño & $\mathbf{1 6}$ \\
\hline TOTAL &
\end{tabular}


Se procedió a realizar una búsqueda de términos clave en inglés encontrándose 14 resultados, tal como se observa en la Tabla 4. Los más numerosos fueron encontrados bajo el concepto de solución de problemas de diseño. Vale la pena considerar que los registros más trabajados fueron aquellos que tenían el concepto de "diseño" relacionado con "problemas" en inglés y español. En total se consolidaron 30 registros.

Tabla 4. Resultados obtenidos por palabras clave en inglés.

\begin{tabular}{ll}
\hline Palabras clave en ingles & \\
\hline Problem Solving & 3 \\
Solve design problems & 5 \\
Problem solving and cognitive styles & 1 \\
Design problem solving & 5 \\
\hline TOTAL & $\mathbf{1 4}$ \\
\hline
\end{tabular}

Con este sistema de búsqueda se obtuvieron artículos con aportes significativos y como puede verse en la Gráfica 1, es posible apreciar una especie de ritmo de crecimiento en la cantidad de publicaciones a razón de un artículo cada dos años.

Figura 2. Año de las publicaciones.

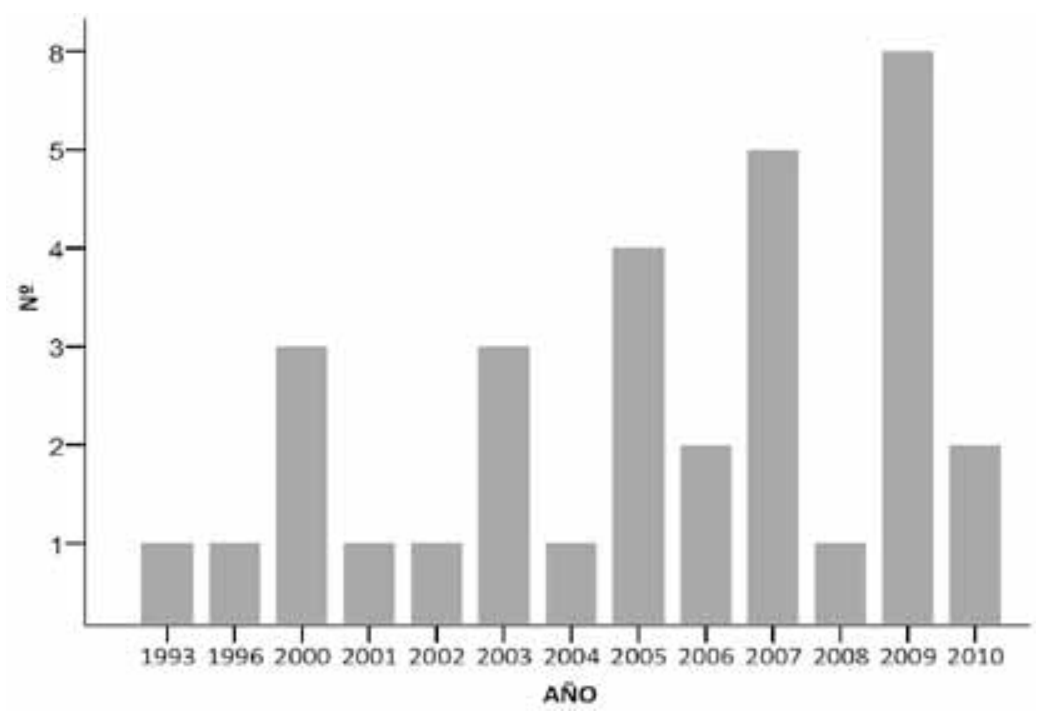

No obstante, el número de producciones es un tanto restringido para un periodo de 17 años, por lo que de antemano cabe destacar la importancia de continuar con la investigación sobre este ámbito de la tecnología, el diseño y la pedagogía desde la perspectiva de la CPS. 


\section{b. Bases de datos consultadas}

A continuación se muestran los resultados obtenidos en función de las bases de datos en donde se encontraron los artículos indexados sobre formulación y solución de problemas en diseño y tecnología.

Figura 3. Resultados por cada base de datos consultada.

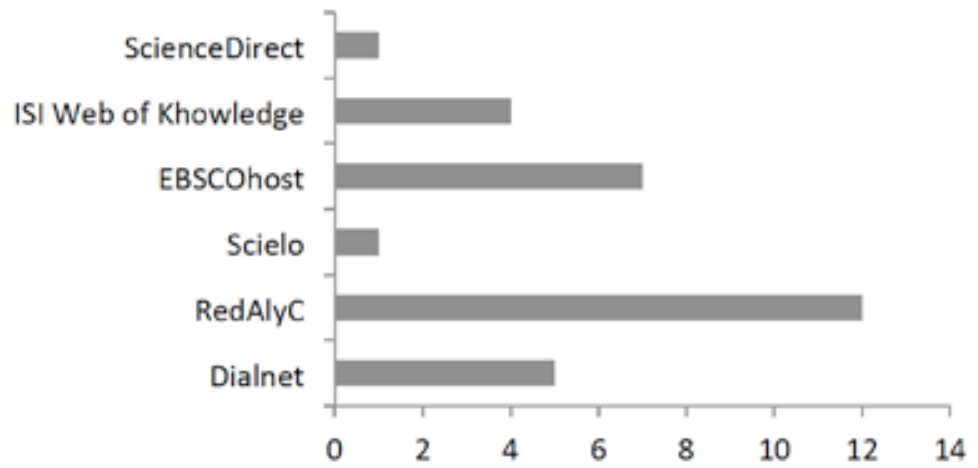

De acuerdo con la Figura 3, la distribución de artículos muestra mayor concentración en la Red de América Latina y el Caribe (REDALyC), en segundo lugar EBSCOhost con 7 resultados, luego Dialnet con 5 artículos; finalmente, en ISI se presentaron 4. En último lugar, aparecen simultáneamente ScienceDirect y Dialnet con un resultado cada una. Como se verá en la Figura 4, la cual da cuenta del país de origen de cada publicación, quienes más emplean estas bases de datos para divulgar sus resultados de investigación son Estados Unidos, en primer lugar, y, en segundo lugar, Colombia. En tercer lugar aparecen Australia, México y Holanda con tres publicaciones cada uno. Luego siguen Inglaterra y Argentina con 2 artículos cada uno. Finalmente, Canadá, Corea, Alemania, España, Israel y Japón presentan cada una 1 publicación.

Figura 4. Resultados por país de la procedencia de los artículos.

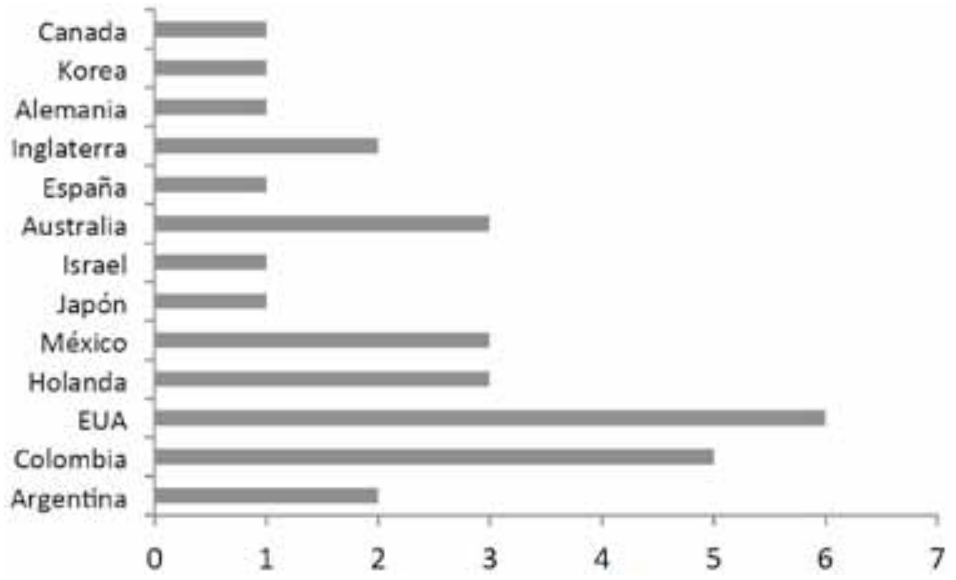

La producción especializada presentada, pone de relieve la necesidad de seguir explorando estos conceptos con esfuerzos en más investigaciones, dada su importancia para la compresión de los procesos de diseño, la formación para que sean cada vez mejores y más eficientes las respuestas creativas y productivas que promuevan el desarrollo de la sociedad y el medio ambiente. 


\section{c. Clasificación de las referencias por temas tratados}

Ahora se presentan los resultados organizados por los temas vinculados con la formulación y solución de problemas en diseño y tecnología. Los aportes más numerosos en la revisión son aquellos interesados en abordar el tema desde el ámbito pedagógico y didáctico, le siguen las ciencias cognitivas, el enfoque por competencias y en menor cantidad las TICs, la ingeniería, las reflexiones sociológicas sobre problemáticas CTS y el campo del diseño. Así puede verse a continuación en la tabla 5.

Tabla 5. Principales aspectos tratados en los artículos.

\begin{tabular}{ll}
\hline Tema & $\mathbf{N}^{\mathbf{0}}$ \\
\hline Competencias & 3 \\
Didáctica & 7 \\
Pedagogía & 8 \\
TICS & 2 \\
Ingeniería & 2 \\
Diseño & 2 \\
Cognición & 4 \\
CTS & 2 \\
\hline TOTAL & $\mathbf{3 0}$ \\
\hline
\end{tabular}

A continuación se presentan los resultados de la revisión documental dando cuenta de los distintos campos de conceptualización y sus aportes para el campo del diseño.

\section{Distintos campos de conceptualización sobre formulación y solución de problemas}

\section{a.Psicología Cognitiva y educación en tecnología}

La formulación y solución de problemasse define como una capacidad cognitiva y física del individuo para presentar alternativas a situaciones que presentan algún grado de desequilibrio (Piaget, 1981) o desajuste. Una de las teorías fundamentales que desarrolla estos conceptos dentro del campo de la educación en tecnología son los trabajos de Andrade y sus colaboradores $(1993,1996,1998)$. Ellos exponen los atributos que enmarcan patrones bajo los cuales se caracteriza los ambientes tecnológicos de tarea, la estructuración del espacio del problema de diseño y la presentación incremental de alternativas de solución. A continuación, se presentan brevemente algunos de los elementos estructurales característicos del proceso que lleva de la formulación hasta la solución de problemas de tecnología y diseño: 
- Ambiente de tarea, a modo de situaciones problemáticas, definidas en el diseño por expectativas, necesidades o situaciones problemáticas, desajustes y desequilibrios en contextos específicos. Tratados desde el ámbito profesional del diseño industrial.

- Proceso de Negociación de la construcción y estructuración del espacio de problema de modo no técnico entre el diseñador y los actores involucrados.

- Ciclos recurrentes en el proceso de estructuración de la formulación del problema y de la solución.

- Dimensiones relacionales de la solución de problemas entre lo holístico - particular como modalidades de aproximar a la solución mediante la toma de decisiones argumentadas. No relacionado con metodologías de diseño(Andrade \& Lotero, 1998). Existen evidencias que señalan que los estudiantes al seguir determinados procesos de diseño realizan tareas secuenciales sin continuidad y no llegan a construir una visión compleja y sistémica del proceso de diseño(Osorio, 2008; Cross, 2002).

- La experiencia previa del diseñador es el elemento que le permite argumentar y elegir las rutas más probables de las alternativas que se exploran, especializan y evalúan en esa elección. Vincula las estrategias de solución de problemas como procesos heurísticos - algorítmicos(Boden, 1994; Perkins, 1989; Dahlman, 2007).

- La solución final (temporal) como síntesis incremental del artefacto tecnológico, comprobada de las alternativas que se exploran, especializan y evalúan en esa elección. La presencia de pensamiento divergente operaría con altos niveles de abstracción (Novak, 1995), y conlleva en palabras de Boden, el dominio de las "reglas y restricciones", (una especie de juego, ej.: (Parra, 1996:100) y pasaría por procesos mentales como Incubación, maduración, etc. (Boden, 1994).

- Estrategia de control de compromiso limitado que por lo general se desarrolla y estructura a partir de una alternativa inicial (Goel\&Pirolli, 1992) pero se abandona en determinado tiempo.

- La solución de diseño no es únicamente lógica sino que presenta unas inferencias no deductivas derivadas de un pensamiento supraordenado (Novak, 1995). Ausubel, plantea que:"un fundamento para la presentación de soluciones debe tener como base la construcción de estructuras previas sobre las cuales hacer inferencias para llegar a un nivel supraordenado que le permite ser creativo en el planteamiento de la solución" (Ausubel, 1990).

Figura 5. Proceso de estructuración que lleva a la formulación y a la solución incremental del problema.

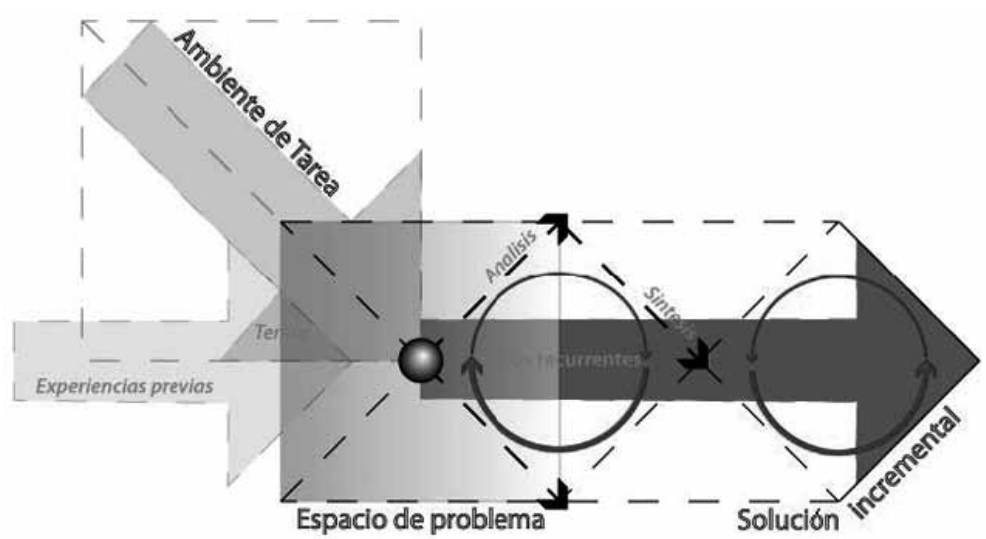


No puede dejarse de lado la distinción de la tecnología como una estructura del mundo de la vida que en palabras de Vargas (1999) desde la línea de Heidegger se refiere a:

"una representación del mundo, que tiene a la base el diseño de relaciones sociales, económicas, culturales, educativas junto con condiciones generadoras de conocimientos y productos a partir de la resolución de problemas en diferentes ámbitos de la vida cotidiana, tanto en su dimensión científica como cultural.” (Vargas: 136).

En este sentido la tecnología tiene repercusiones claras para el desarrollo individual y social, por lo tanto ésta impone un enfoque que involucra lo pedagógico (formación del sujeto), educativo (reflexión acerca del tipo de sociedad y calidad de vida) y lo epistemológico, en tanto generadora de conocimientos a partir del diseño. Como consecuencia Vargas (1999) recurre a Antoni Colom para señalar que:

"ello presupone afirmar que una educación para el cambio implica profundizar en la individualización, ya que las soluciones adaptativas se encuentran en los propios recursos mentales e intelectuales del sujeto.” (Ibíd: 137).

\section{b. Una delimitación necesaria acerca del Diseño Industrial}

En la mirada del filósofo Gastón Bachelard, el diseño es una región epistemológica en donde lo teórico se transforma en materialidad (Bachelard, 1994:15-26). Este hace las veces de un operador que transforma la abstracción en concreción. Y de acuerdo con Carlos Federici, el diseño corresponde a una prefiguración inteligible de lo concreto desde el signo escrito(Gallego, 1995).

Así, el Diseño es un escenario que problematiza en el aspecto epistemológico las interrelaciones sistémicas complejas con lo cultural en los aspectos antropológico, psicológico y sociológico. Busca que en la estructura cognitiva del diseñador desarrolleevolucione, esto requiere generación de reglas lógica-creativas y de organización de la experiencia y el conocimiento discursivo. Así mismo, de las interacciones personales, disciplinares, inter y transdisciplinares(García, 2006; 2000). Esto conlleva a la modelación mental de un propósito en y para la transformación cualitativa de un contexto social, cultural y ambiental. (Andrade \& Lotero, 1998; Martínez E. , 2006).

Este desarrollo creativo requiere, como lo plantea Norbert Wiener(1995), cuatro climas para su proyección: el clima intelectual (Científico-tecnológico), el clima técnico (productivoconformativo), el clima social (humano-cultural) y el clima económico (beneficio-costo). En esta nueva red compleja de interacción de variables estructuradas por el diseñador en el proceso de diseño, evidenciará la jerarquía cognitiva y su proposición innovadora, compleja y sistémica en el contexto socio-cultural. 
Este tejido conceptual nuevo logrado a partir de su malla cognitiva, es lo que denomina Novak(1995) conocimiento supraordenado: aquel que es organizado y jerarquizado en altos niveles de abstracción, combinación e inclusividad.

\section{Aspectos cognitivos en el proceso de formulación y solución de problemas en diseño}

El enfoque que se deja ver en el proceso de diseño va de la estructuración de un escenario de intervención hasta la presentación final de un producto de diseño, está enmarcado en una construcción de proyecto. Epistemológicamente, el enfoque más pertinente es el psicogenético y constructivista, trabajados ampliamente por Piaget (1994) y desde otra arista por Vigotsky $(1995 ; 1996)$. Con esta perspectiva onto y socio genética se entiende que el diseño atraviesa por procesos psicológicos superiores, formas de organización mental (Piaget, 1994; Piaget, 1981) e interiorización cultural por parte del individuo (Vigotsky, 1996; Vigotsky, 1995). Esto se puede visualizar de manera dinámica y progresiva en los procesos de diseño que emprenden profesionales y estudiantes.

García (2000) aclara que estas formas organizativas requeridas en los procesos de diseño y creación llevan a la construcción de conocimiento y menciona que el desarrollo del conocimiento consiste en un doble proceso. A su vez, Vargas Guillen (1999) no duda en señalar esto como un nuevo espacio para la generación de conocimiento a partir del diseño de soluciones a problemas concretos dentro del marco de la condición postmoderna. Siguiendo a García (2000) en este proceso se identifican dos elementos indispensables:

\section{- La organización de las propias actividades del sujeto}

"Que comienza con la coordinación de sus acciones, continua con el desarrollo de los mecanismos constructivos del conocimiento, y culmina con la lógica, es decir, en las formas deductivas y los reforzamientos.” (2000: 112).

\section{- La organización del material empírico}

"Que comienza con asignación de significados, continua con comparaciones que conducen a correspondencias y transformaciones elementales y culmina en la interpretación de fenómenos estableciendo relaciones causales." Ibíd.

García concluye mencionando que estos desarrollos conducen a obtener preguntas de carácter epistémico por lo que se refuerza la manera particular de construcción de conocimiento de la tecnología y el diseño que atraviesa estados de apropiación intelectual que conducen a estados de consciencia cada vez más organizados. Esto pone de relieve su carácter como cuerpo discursivo y disciplinar.

En este sentido, se hace necesario establecer cómo es el proceso que lleva a la toma de conciencia de la existencia de un problema que, en los ojos de lego, nunca lo sería (Piaget, 1981). Como lo señala Hanson, citado por García: “el niño y el profano pueden ver: ellos no son ciegos. Pero ellos no pueden ver lo que ve el físico: ellos son ciegos con respecto a lo que éste ve." (2006: 41).

Por su parte Gallego pone de manifiesto que las construcciones en el conocimiento, 
es decir, en la construcción de un problema que organiza las experiencias en conceptos (entendiendo los problemas como constructos intelectuales que implican solución mediante tareas de diseño), no pudieron ser azarosas y este desarrollo requiere de reflexiones que separen la intuición. Se trata de ir más allá de la pregunta por el cómo para preguntarse el porqué de los fenómenos. Es así que al mencionar a N.H. Coghlan explica los logros que consolidaron la etapa técnica de las civilizaciones; Gallego se refiere a "una serie de conquistas que no son explicables aduciendo acumulación fortuita y hallazgos al azar.” (1995:73).

Parece claro que al asumir los problemas como construcciones, estos estarían inscritos dentro de procesos de los cuales pueden distinguirse las fases o procesos propiamente dichos y las operaciones de apoyo a esos procesos. Respecto a lo primero, Stoyanov\&Kirschner de la Universidad Abierta de Holanda plantean los siguientes procesos (2007, pág. 50).

- Analizar la situación del problema

- Generar ideas

- Seleccionar las ideas más apropiadas

- Implementar y evaluar

En relación con los procesos de apoyo a estas fases en la solución de problemas estos autores plantean la pregunta acerca de:

- ¿Cómo proceder en esas fases de la solución de problemas?

- ¿Qué hacer al analizar la situación del problema?

- ¿Cómo generar las ideas?

- ¿Cómo seleccionar una solución e implementarla en la práctica?

Para tal fin, los autores ponen de manifiesto que estos serían logros deseados dentro de un aprendizaje de competencias ${ }^{11}$, para lo cual vale la pena hacer la relación con el Diseño Industrial como profesión(Álvarez \& Martínez, 2009b; Mazzeo \& Romano, 2007), problemas que estarían determinados por la naturaleza de los "problemas débilmente estructurados" (Goel \& Pirolli, 1992) y las estructuras cognitivas involucradas en procesos para la presentación de alternativas de solución (Stoyanov \& Kirschner, 2007; Kim, Kim, Lee, \& Park, 2007).

Bajo esta misma línea que investiga los procesos de formulación y solución de problemas, Treffinger y colaboradores (2008), desarrollaron un modelo para representar los procesos que requieren de la CPS recogiendo, por más de cinco décadas, variadas investigaciones con diferentes grupos e instituciones entre colegios, universidades, pequeñas y grandes empresas y organizaciones (Treffinger, Selby, \& Isaksen, 2008). 
El modelo de Treffinger sugiere que los procesos de solución de problemas, lejos de ser lineales, se constituyen en núcleos de carácter circular, es decir que se retroalimentan constantemente; aunque desafortunadamente no se encontró cómo Treffinger y colaboradores dan sentido a la propuesta de los núcleos de carácter circular. No obstante, en la propuesta de Andrade y sus colaboradores (Maldonado \& Andrade, 2001; Andrade \& Lotero, 1998) se resuelve con el concepto de "ciclo recurrente" en el proceso de síntesis incremental presentado en párrafos anteriores cuya estructura consta de cuatro elementos fundamentales los cuales incluyen, a su vez, subetapas(Treffinger, Selby, \& Isaksen, 2008: 391-393):

1. Entendiendo el desafío: definir, construir y focalizar los esfuerzos para solucionar el problema.

a. Implica construir oportunidades

b. Exploración de datos

c. Enmarcar el problema.

2. Generando ideas: implica una aproximación, mediante opciones variadas, para dar respuesta al problema.

3. Preparándose para la acción: implica la toma de decisiones para comprometerse con la promesa de una alternativa y planear su implementación.

a. Desarrollo de soluciones

b. Construcción de la aceptación.

4. Planeando el acercamiento: es un componente administrativo que guía a los Diseñadores (solucionadores de problemas) en el análisis y selección de componentes procesales y estrategias deliberadas, (Kim, Kim, Lee, \& Park, 2007).

Como se ha intentado poner de manifiesto, esta estructura descrita brevemente tiene múltiples coincidencias con la propuesta planteada por de Andrade y colaboradores, lo que refleja una coherencia, desde diferentes aproximaciones, en las estructuras y procesos ocurridos en la CPS. El proceso de la estructuración del ambiente de tarea y el paso hacia la definición del espacio de problema de diseño tiene correspondencia con las tareas involucradas en "entender el desafío"planteadas por Treffinger y colaboradores; así mismo, la "síntesis incremental del artefacto" comprendería los pasos de "preparación para la acción” y la "generación de ideas" de Treffinger y colaboradores. Finalmente, los ciclos recurrentes de negociación estarían identificados con la valoración de las tareas y los que podrían llamarse procesos administrativos en diseño.

Otra interesante perspectiva la aporta Schraagen(1993) quien explica cómo los diseñadores expertos resuelven problemas y describe cuatro tipos de conocimiento requerido por los expertos (Schraagen, 1993: 287-289) ${ }^{12}$ : 
- En el área de conocimiento (conocimiento disciplinar, discursivo de diseño).

- De estrategias heurísticas (conocimiento tácito del hacer (Fleer, 2000).

- De estrategias de control (lógica estratégica).

- De estrategias de aprendizaje (aprender de experiencias en la solución de problemas).

Estos cuatro tipos de dominio de conocimiento tienen relación directa con múltiples investigaciones desde la perspectiva del "dominio de conocimiento en diseño"(Andrade \& Lotero, 1998; Bachelard, 1994; Barak \& Goffer, 2002; Cross, 2002; Kolfschoten, Lukosch, Verbraeck, Valentin, \& Vreede, 2010; Meneely \& Portillo, 2005; Álvarez R. \& Martínez S., 2010b,c; DIT, 2009).

Esta consolidación de las miradas a los procesos entorno al problema de diseño permite presentar a continuación, las relaciones entre el proceso que va de la estructuración y formulación de un problema a su solución incremental, relacionada con los EC. Esto, a partir de la manera consistente en que los solucionadores de problemas prefieren aproximarse mediante la realización de actividades mentales y físicas focalizadas(Jonassen D. H., 2000; Kolfschoten, Lukosch, Verbraeck, Valentin, \& Vreede, 2010; Schraagen, 1993; Goel \& Pirolli, 1992; Layton, 1993; Perkins, 1989; Hederich \& Camargo, 1998).

\section{a. Los estilos cognitivos y CPS}

En los resultados encontrados hasta el momento, durante la primera etapa de desarrollo de la investigación sobre competencias para la innovación, se exploró el concepto de los estilos cognitivos (EC); especialmente se trabajó con la dimensión de Sensibilidad - Independencia al campo (SIC) como aquella polaridad del EC en la que según Witkin \& Goodenough (1985), “..., producto de las investigaciones de Witkin y su equipo les llevó a organizarla en una nueva estructura que ya no podría entenderse bajo la tendencia de un esquema de la "personalidad global” (Álvarez R. \& Martínez S., 2010a:13).

Los diferentes hallazgos se estructuraron bajo el concepto de "diferenciación", que se entendió como, “... un constructo útil para conceptualizar el amplio panorama de las consistencias individuales...” (Witkin \& Goodenough, 1985: 41).

Esta dimensión SIC se examinó conjuntamente con los procesos de diseño de objetos de uso cotidiano (Álvarez \& Martínez, 2009b), donde se contemplaron una serie de planteamientos relacionados con los procesos en diseño, por ejemplo: (Spotts \& Mackler, 1976) se han preocupado por la relación entre los EC y la creatividad; sin embargo, difícilmente pudieron concluir que existiera evidencia de la preferencia por alguna polaridad SIC en las tareas creativas, de tal modo que tanto los Independientes al campo como los Sensibles son individuos potencialmente creativos.

Dentro de esta misma conclusión en la enseñanza del diseño, se encontró la necesidad de implementar didácticas incluyentes para los diversos perfiles de estudiantes que promuevan la movilidad de sus polaridades de EC(Bloomberg, 1971; Meneely \& Portillo, 
2005), así como también de los estilos de aprendizaje y los estilos de pensamiento que han desarrollado los estudiantes(Sternberg \& Zhang, 2001). Conviene además, realizar más investigaciones acerca de los estilos de enseñanza de los docentes ${ }^{13}$ (Kolfschoten, Lukosch, Verbraeck, Valentin, \& Vreede, 2010; Turner, 2009; Jonassen D. H., 2000).

No obstante lo anterior, hasta el momento el tema de la solución de problemas lleva en realidad un proceso completo de estructuración, formulación y solución incremental de problemas. En algunas investigaciones relacionadas, puede observarse una aproximación integral de estos procesos involucrados en lo que se conoce como solución creativa de problemas (CPS), aproximación teórica que cuenta con una amplia exploración de más de cinco décadas de investigación (Treffinger, Selby, \& Isaksen, 2008).

En relación con los procesos que ocurren en la solución de problemas es importante hacer alusión al trabajo de Johnson (1972:133) citado por (Treffinger, Selby, \& Isaksen, 2008) quien se centra en el problema como: un vacío entre ¿donde se está? o, ¿qué se tiene? y una posición deseada o resultado.

En detalle Treffinger et al.plantean que los estilos influyen en cómo los individuos perciben la información y los problemas, procesan datos, preparan e implementan la solución, también como usan constructivamente la información para solucionar problemas y manejar los cambios más efectivamente. ${ }^{14}$

Es oportuno insistir en que una buena aproximación al enfoque de los EC en la solución de problemas lo proporciona Treffinger y colaboradores, quienes los definen como:

“diferencias individuales consistentes en la manera en que las personas prefieren planificar para llevar a cabo actividades focalizadas, con el fin de obtener claridad, generar ideas y prepararse para la acción. Una disposición natural de los individuos para manejar los cambios y solucionar problemas influenciada en parte por el modo de pensar, la motivación para tomar parte y responder a una situación que se le ha presentado, y las dimensiones actitudinales de su personalidad". ${ }^{15}$

Treffinger y colaboradores concluyeron al igual que otros estudios(Bloomberg, 1971; Meneely \& Portillo, 2005; Álvarez \& Martínez, 2009a), que las dimensiones de los estilos en la solución de problemas son polaridades potencialmente creativas y no puede haber preferencias por una u otra, todos los estilos juegan un papel importante en la vía de la solución de problemas. 
En cuanto al modelo Treffinger y colaboradores plantean que este implica el pensamiento y el comportamiento en la consecución de un objetivo (Jonassen D. H., 2000). Para ello se basan en el proceso de la solución de problemas basada en A.F. Osborn (1952; 1953). Estos autores ponen de manifiesto que dentro de la compresión de la creatividad muchos investigadores vinculan la capacidad creativa de alto nivel con un estilo creativo. Esencialmente los estilos creativos responden a la pregunta ¿cómo se es creativo?

\section{b. Polaridades de los EC relativas a CPS}

Tanto Witkin y colaboradores como Hederich y colaboradores, coinciden en afirmar que la dimensión más holística que es un fuerte indicador del EC es la bipolaridad Sensibilidad-Independencia SIC al campo.(Witkin \& Goodenough, 1985; Hederich \& Camargo, 1998).

Para establecer aspectos relacionados con el EC, en cuanto a las dimensiones SIC es pertinente mencionar lo señalado por Witkin \& Goodenough (1985) cuando se refieren a esta dimensión, como la que engloba cuestiones de la percepción y a su vez de la personalidad individual bajo el argumento de la psicología diferencial que trasciende a los EC. En general:

"las razones por las que existe mayor inversión investigadora sobre la dependencia-independencia de campo en contraste con otros estilos cognitivos, son numerosas y diferentes. Entre estas razones está la demostrada amplitud de la dimensión y su evidente representación en la vida diaria, de tal forma que sus manifestaciones son notorias...” (Witkin \& Goodenough, 1985:25).

Aunque las polaridades se constituyen en un todo integral del individuo, existen específicamente unas polaridades de los estilos estrechamente vinculadas con CPS que se muestran de acuerdo con las fases de formulación y solución de problemas en la Tabla 5. Vale la pena distinguir entre la solución de problemas con la creatividad en el sentido de la creación de nuevas ideas relativa a la creatividad, mientras que la solución de problemas obedecería a la obtención de un propósito no necesariamente de manera original o novedosa.

A continuación, se especifican las polaridades relacionadas con la CPS desde la perspectiva de los estilos en la solución de problemas: 
Tabla 6. Principales polaridades de los estilos cognitivos en la CPS. Elaborada por los autores.

\begin{tabular}{|c|c|c|}
\hline Autor & Dimensión del estilo & Descripción \\
\hline \multirow[t]{3}{*}{$\begin{array}{l}\text { (Treffinger, Selby, \& Isaksen, } \\
2008)\end{array}$} & $\begin{array}{l}\text { Explorador vs Desarrollador } \\
\text { Orientación al cambio }(\mathrm{OC})\end{array}$ & $\begin{array}{l}\text { Los exploradores tienden a solucionar los problemas desde } \\
\text { la perspectiva de vistalizar posibilidades inustales, explora } \\
\text { nuevas vias y posibilidades; por el contrario los } \\
\text { desarrolladores enfrentan los problemas a partir de algunos } \\
\text { elementos básicos iniciales, soluciones prácticas y con la } \\
\text { realidad de la tarea. }\end{array}$ \\
\hline & $\begin{array}{l}\text { Interno vs. Externo o } \\
\text { Introversión vs extroversión (Meneely \& } \\
\text { Portillo, 2005) } \\
\text { Modo de procesamiento (MP) } \\
\text { (caracteristicas similares a la dimensión } \\
\text { independencia-dependencia al campo } \\
\text { (Álvarez R. \& Martinez S., 2010: Hederich } \\
\text { Martinez, 2004) }\end{array}$ & $\begin{array}{l}\text { Los externos son personas que tienden a solucionar } \\
\text { problemas desde la interacción activa con otros discutiendo } \\
\text { las ideas: por el contrario los internos hacen gala de sus } \\
\text { propios recursos para tomar decisiones en la solución de } \\
\text { problemas. }\end{array}$ \\
\hline & $\begin{array}{l}\text { Centrado en las personas vs. Centrado en } \\
\text { las tareas } \\
\text { Vias de decisión (WD) }\end{array}$ & $\begin{array}{l}\text { Los individuos centrados en las personas consideran } \\
\text { primero el impacto de sus decisiones en las otras personas. } \\
\text { Prefieren estar involucrados emocionalmente cuando } \\
\text { establecen prioridades. }\end{array}$ \\
\hline \multirow{2}{*}{$\begin{array}{l}\text { Indicador de tipos de Myers Briggs } \\
\text { (MBTI) citado en: (Meneely \& } \\
\text { Portillo, 2005, paig. 159; Pantoja } \\
\text { O., 2005) } \\
\text { Kagan J. citado en: (Hedereich \& } \\
\text { Camargo, 1998, pág. 32) }\end{array}$} & Intuición us sensación & $\begin{array}{l}\text { Los intuitivos responden a situaciones basados en la } \\
\text { inconsciencia ya que generalmente no pueden explicar sus } \\
\text { decisiones; mientras que las personas sensitivas tienden a } \\
\text { resolver problemas reaccionando a estimulos. }\end{array}$ \\
\hline & $\begin{array}{l}\text { Pensamiento vs emoción } \\
\text { o reflexividad vs impulsividad' }\end{array}$ & $\begin{array}{l}\text { Las personas prefieren decidir rápidamente (pensamiento } \\
\text { impulsivo) con poca probabilidad de certeza. Por el } \\
\text { contrario los pensadores consideran los escenarios antes de } \\
\text { dar la respuesta controlando el error (pensamiento } \\
\text { reflexivo). }\end{array}$ \\
\hline $\begin{array}{l}\text { (Kirton, 2003) citado en: (Stoyanov } \\
\text { \& Kirschner, 2007; Pantoja O., } \\
\text { 2005) }\end{array}$ & Adaptador vs innovador & $\begin{array}{l}\text { Los adaptadores tienden a adherir elementos a una } \\
\text { estructura establecida, mientras que los innovadores tienden } \\
\text { a resolver problemas sin una estructura particular más } \\
\text { inusuales pero menos factibles. }\end{array}$ \\
\hline $\begin{array}{l}\text { Bruner J., citado en: (Hederich \& } \\
\text { Camargo, 1998, pág. 31) }\end{array}$ & Centrado vs Barrido & $\begin{array}{l}\text { Los individuos tendientes a la centración se enfocan en una } \\
\text { sola tarea y la finalizan antes de iniciar otra, mientras que la } \\
\text { tendencia al barrido implica realizar varias tareas al tiempo } \\
\text { sin importar el final. }\end{array}$ \\
\hline $\begin{array}{l}\text { (Hederich, 2004; Blanca Mena \& } \\
\text { Luna Blanco, 1990; Bloomberg, } \\
\text { 1971: Iriarte, Cantillo, \& Polo, } \\
\text { 2000; Meneely \& Portillo, 2005; } \\
\text { Spotts \& Mackler, 1976; Witkin, } \\
\text { Moore. Goodenough. \& Cox. 1977) }\end{array}$ & Independencia vs dependencia & $\begin{array}{l}\text { Desarrollada previamente ea la primera fase del proyecto } \\
\text { (Álvarez \& Martinez, 2009). Dimensión bolistica que } \\
\text { puede abarcar otras polaridades. }\end{array}$ \\
\hline
\end{tabular}

Es importante Acotar lo señalado por Treffingeret al. ${ }^{16}$, cuando advierten sobre la importancia de estas distinciones a nivel de los estilos de solución de problemas ya que existen prejuicios acerca de la creatividad, por lo general la asociación con algunas profesiones como las artísticas; también se tiende a desconocer la tarea creativa de los ingenieros (2008:396). Lo anterior puede llevar a los programas educativos a privilegiar didácticas para unos y excluir el desarrollo creativo en otros.

Como lo menciona Kirton (2003), citado por Stoyanov \& Kirschner (2007), los EC tienen un "valor neutral" y cada polaridad o dimensión del estilo puede producir soluciones creativas a problemas, tal como lo confirman sus hallazgos. Así mismo, las investigaciones de Kirton sugieren que las personas pueden operar con diferentes dimensiones del estilo en cada una de las etapas del proceso de solución de problemas.

En algunos estudios puede encontrarse algunas críticas a la relación entre las dimensiones de los EC y su posibilidad de explicar algunos procesos de CPS. No obstante, esto puede ser atribuido tanto a la metodología experimental como a la concepción o enfoque acerca de los EC. ${ }^{17}$ Por ejemplo, es importante destacar que algunos hallazgos de investigaciones conceptualizan la diferencia entre los EC de constructos cognitivos tales como conocimiento e inteligencia (Kirton; 2003, citado por Stoyanov \& Kirschner (2007:53). 
Finalmente, es pertinente hacer referencia a Pantoja quien citando a Cross N. (1991), reconocido investigador en el campo del diseño, expone la estrecha relación entre la actividad de diseño, especialmente el proceso para formular, estructurar y resolver problemas de manera creativa:

"la forma como el individuo aborda la situación, que se convierte en su objeto de conocimiento, y la forma como plantea soluciones para conocerlo mejor o para solucionarlo, depende de su estilo particular o estilos predominantes" (Pantoja O., 2005).

\section{Cometarios finales}

La necesidad que mueve el desarrollo de la presente investigación implica aportar, desde una perspectiva cognitiva, aproximar y sugerir algunas estrategias didácticas para la disciplina del diseño (Dahlman, 2007; Kim, Kim, Lee, \& Park, 2007; Jonassen \& Hernandez Serrano, 2002), que, en alguna medida, beneficien los procesos de formación de diseñadores industriales.

El (PEI) de la universidadpromueve una educación incluyente y por ello se hace necesaria la adecuación de las prácticas educativas desde la pedagogía y la didáctica. Estas se desarrollan mediante la construcción de conocimientos sobre contenidos, la enseñanza y el aprendizaje. Las reflexiones que se esperan obtener de estas reflexiones también podrán servir, en alguna medida, como fuente de consulta para otros programas académicos de la universidad o externos interesados en la comprensión de los aspectos cognitivos implicados en la solución creativa de problemas y su relación con las competencias para la innovación.

Maldonado y Andrade (2001) han definido algunas condiciones para la educación de los individuos para que puedan resolver problemas con el fin de desarrollar lo que denominan como "la capacidad de diseño". En este sentido, la presente propuesta recoge los aspectos diferenciales cognitivos y los relaciona con los disciplinares que construyen el objeto de estudio del diseño como profesión. 
Para construir una propuesta en este campo, es importante revisar las condiciones que Maldonado y Andrade exponen:

1. Desarrollo de ciertas habilidades y destrezas tanto motrices como de expresión y experimentación.

2. Una malla conceptual a modo de una experiencia mínima como base para un aprendizaje significativo (Dahlman, 2007).

3. Capacidad de expresar, en términos abstractos (capacidad de pensamiento abstracto), los referentes concretos como situación de partida para la tarea de diseño.

4. Construcción de prototipos funcionales que expresen, la estructura y la función de las soluciones, parciales o finales, a los problemas de diseño.

5. Experiencia significativa del individuo. "La construcción de estrategias fuertes de solución de problemas, que es el aspecto que diferencia la labor de los especialistas de los novatos" Gagné (1984), citado por Maldonado \& Andrade, (2001:19); Schraagen, 1993; Liikkanen \& Perttula (2008).

6. Estudio de la historia del desarrollo de los objetos. El estudio sistemático de las soluciones prácticas conociendo la experiencia técnica de la humanidad.

7. Partir del aprendizaje por descubrimiento guiado para culminar en aprendizaje por descubrimiento autónomo.

Puede resultar casi paradójico enseñar a diseñar si se considera esta una capacidad innata en los individuos, que además implica pensamiento divergente en tanto que los procesos académicos generalmente son "frontales" y convergen en resultados a veces homogéneos. Como se ha visto, las complejidades que se requieren para el procesamiento y las estrategias implicadas en la estructuración de un proyecto de diseño que conlleve al planteamiento de una idea original e innovadora, es decir que se lleve a su fabricación o puesta en el uso cotidiano, implica el trabajo de más de un individuo, ya que estos procesos proyectuales desbordan en información, y requieren optimización del tiempo, un panorama que seguirá siendo un reto en los programas educativos de diseño que desconocen el elemento grupal y colectivo del diseño y la innovación.

No obstante, el diseño visto desde una perspectiva compleja y sistémica permite entender que los hallazgos de las investigaciones, aunado a los esfuerzos pedagógicos y didácticos puestos en las aulas, permiten articular sincrónicamente y de modo jerárquico, muchos de estos componentes complejos de la ecuación "formación en y para el diseño". Por otra parte, en la academia se encuentra el reto de pensar epistemológicamente y evaluar críticamente las posibilidades de formación de personas cada vez más creativas, poniendo en la palestra los componentes para una educación incluyente, que respete la diversidad de las personas y sus potenciales. ${ }^{18}$ 
1. Origen del documento: Álvarez, F. y Martínez, E. (2009). Identificación del estilo cognitivo del estudiante de Diseño Industrial de la UJTL. Informe de investigación. No. Registro 180-04-2008, financiado por la Dirección de investigación de la Universidad Jorge Tadeo Lozano.

2. Magíster en Pedagogía de la tecnología de UPN (2003), Diseñador industrial UJTL (1996). Profesor Asociado del programa de Diseño Industrial en la Universidad Jorge Tadeo Lozano. Tel.2427030 ext.1739.cel.3124322973.fernando.alvarez@utadeo.edu.co.

3. Magíster en Pedagogía de la tecnología de Universidad pedagógica Nacional (2001). Licenciado en Diseño Tecnológico UPN (1991), Profesor Docente Magíster del programa de Diseño Industrial en la Universidad Jorge Tadeo Lozano.Tel.2427030 ext.1739.edgar.martinez@utadeo.edu.co. 4. La sustitución del concepto de "dependencia al campo" por el de "sensibilidad al campo" ha sido abordada en el sentido de la acepción positiva que tiene el ser sensible, puesto que en las explicaciones que se les ofrecen a los estudiantes, sujetos del estudio, tiende a ser tomado negativamente la palabra dependencia y no corresponde con la bipolaridad que tiene la dimensión. Éste ha sido discutido también por Ramírez y Castañeda (1974), referenciados por Hederich, C., \& Camargo, A. (1993:36).

5. Por ejemplo, Hederich y Camargo, 1993, 1999; Hederich, Camargo, Guzmán y Pacheco, 1995; Iriarte, Cantillo, \& Polo, (2000).

6. Hederich, Camargo, \& Reyes, Ritmos Cognitivos en la Escuela, (2004:48).

7. En detalle se puede estudiar la validez de la prueba en: Hederich, (2004:259-262); quien en su tesis doctoral presenta la consolidación de 20 años de trabajo sobre la línea de los estilos cognitivos.

8. Por sus siglas en inglés CreativeProblemSolving (CPS) (Treffinger, Selby, \& Isaksen, 2008).

9. Denominado por Hederichet al., como el "modelo frontal individualizado" (.

10. Búsquedas realizadas por los investigadores y la colaboración de la estudiante María Fernanda Ángel del programa de Diseño industrial de la Tadeo y la colaboración de la diseñadora Carolina Parra.

11. El concepto de competencia es reevaluado por los autores y puede verse ampliamente en: Álvarez, F., \& Martínez, E. (2010). Competencias para la innovación: Identificación de competencias cognitivas significativas del profesional de diseño. Actas de Diseño, 5.

12. "Domain konwledge, heuristic strategies; control strategies, learning strategies." (cf., 1993:287).

13. Una de las conclusiones del II foro pedagógico de los estilos de aprendizaje organizado por la Universidad de La Salle, ponencia de Christian Hederich; noviembre 4 y 5 de 2009, Universidad de la Salle- Bogotá.

14. "Each dimension influences directly the ways people perceive problems and information, process data, generate possible solutions, make choices and decisions, and prepare to implement solutions. They also provide information that individuals can use constructively to solve problems and manage change more effectively”. (Treffinger, D., Selby, E. C., \&Isaksen, S. G.; 2008:390-401).

15. "We define problem-solving styles as consistent individual differences in the ways people prefer to plan and carry out generating and focusing activities, in order to gain clarity, produce ideas, and prepare for action.” (Ibíd., pág. 393).

16. También revisado por: (Pantoja O., 2005).

17. Hederich y Camargo señalan que la dimensión Holístico vs Analítica postulada por Riding R. como una síntesis de las dimensiones Independencia vs dependencia, reflexividad vs impulsividad, Agudización vs Nivelación y adaptación vs innovación.

18. "..., we focus on the behavioral preferences in solving problems or managing change rather than identifying a general personality type”. Ibid. 395.

19. "The inconsistency of the data related to cognitive style can be attributed to the difference in definition of cognitive style: (a) either as a level-type construct (some styles are better that others), or (b) as a preference to approaching problems in a particular way". (Stoyanov \& Kirschner, 2007:53) 20. Un agradecimiento especial a Fabiola Cabra Torres, $\mathrm{PhD}$ en innovación educativa, docente de la Universidad Javeriana. 


\section{Referencias}

Álvarez, F., \& Martínez, E. (2009a). Caracterización del estilo cognitivo del estudiante diseño industrial de la U.J.T.L. Bogotá: Universidad Jorge Tadeo Lozano.

(10 de octubre de 2009b). http:/ /www.disenola.org. Recuperado el 10 de 10 de 2009, de http:/ / www.disenola.org/index.php/articulos.html?page=shop.product_details\&flypage=flypage.tpl\&product_id=21\&category_ id=10\&manufacturer_id=32

(2010a). Los estilos cognitivos en la dimensión Sensibilidad-Independencia al Campo (SIC) en los procesos de diseño (Vol. 1). Bogotá: Universidad ICESI.

Álvarez R., F. \& Martínez E. (19 de Noviembre de 2010b). Foro de Diseño Industrial. (U. N. colombia, Productor) Recuperado el 3 de diciembre de 2010, de Foro Desconcentrar el diseño: http: / /aplicaciones.virtual.unal.edu.co/blogs/ forodisenoindustrial/articulos

(2010c). Competencias para la innovación: Identificación de competencias cognitivas significativas del profesional de diseño. Actas de Diseño , 5.

Andrade, E. (1993). El papel de la educación en tecnología en el desarrollo nacional de los países del tercer mundo. Bogotá, Colombia: CIUP. (1996). Ambientes de aprendizaje para la educación en tecnología. Revista educación en tecnología, 1 (1), 1-15.

Andrade, E., \& Lotero, A. (1998). Una propuesta de estructura curricular para el desarrollo del área de tecnología e informática. Revista Educación en Tecnología, 3 (3), 72-93.

Ausubel, D. (1990). Psicología educativa: un punto de vista cognoscitivo. Trillas.

Bachelard, G. (1994). La formación del espíritu científico. México: Siglo XXI.

Barak, M., \& Goffer, N. (2002). Fostering systematic innovative thinking and problem solving: Lessons education can learnfrom industry. Interantional Journal of Technology and Design Education (12), 227-247.

Blanca Mena, M. J., \& Luna Blanco, R. (1990). Procesamiento analítico versus holístico en la dependencia-independencia de campo. $8^{\circ}$ Congreso Nacional de Psicología, (pág. 98). Barcelona.

Bloomberg, M. (1971). Creativity as related to field independence and mobility. Journal of Genetic Psychology, 118 (1), 3-12.

Boden, M. (1994). La mente creativa: Mitos y mecanismos. Barcelona: Gedisa.

Cabra, F. (2008). Evaluación de las competencias en la educación superior. Bogotá: Universidad Javeriana.

Carbonell, J. (2006). La aventura de innovar, el cambio en la escuela (III ed.). Madrid, España: Morata.

Cross, N. (2002). Metodos de diseno. Barcelona: Limusa.

Dahlman, Y. (2007). Towards a theory that links experience in the arts with the acquisition of knowledge. JADE, 26 (3), $274-284$.

DIT, D. f. (2009). BIS. Recuperado el 28 de 07 de 2009, de DIT: http: / /www.berr.gov.uk/

Fleer, M. (2000). Working Technologically: Investigations into How Young Children Design and Make During Technology Education. International Journal of Technology and Design Education (10), 43-59.

166 Gallego, R. (1995). Discurso constructivista sobre las tecnologías. Bogotá: Libros y Libres S.A.

García, R. (2000). El conocimiento en construcción. Barcelona: Gedisa. . (2006). Sistemas complejos. Barcelona: Gedisa S.A.

Goel, V., \& Pirolli, P. (1992). Structure of design problem spaces. Cognitive Science, 16 (3), 395-429.

Hederich, C., \& Camargo, Á. (1993). Diferencias cognitivas y subculturas en Colombia. Bogotá, Colombia: Universidad Pedagógica Nacional - CIUP.

Hederich Martínez, C., \& Camargo Uribe, Á. (1995). Logro educativo y estilo cognitivo en Colombia. Revista Colombiana de Educación (30), 48-62.

Hederich Martínez, C., Camargo Uribe, A., Guzmán Rodriguez, L., \& Pacheco Giraldo, J. C. (1995). Regiones Cognitivas en Colombia. Bogotá: Universidad Pedagógica Nacional.

Hedereich, C., \& Camargo, Á. (1998). Estilos Cognitivos como Modalidades de Procesamiento de la Información. Bogotá, Colombia: Universidad Pedagógica Nacional - Colciencias.

Hederich, C., Camargo, Á., \& Reyes, M. (2004). Ritmos Cognitivos en la Escuela. Bogotá: Universidad Pedagógica

Nacional, DGP-CIUP.

Hederich, C. (2004). Estilo cognitivo en la dimensión de Independencia - Dependencia de Campo. Tesis doctoral, Universidad Autónoma de Barcelona, Psicología Basica, evolutiva y educación, Ballaterra, España.

Hederich, C., \& Camargo, Á. (2008). Logro educativo y estilo cognitivo en Colombia. Recuperado el 02 de 11 de 2009, de www. pedagogica.edu.co/storage/rce/articulos/rce30_09infor.pdf

Iriarte, F., Cantillo, K., \& Polo, A. (2000). Relación entre el nivel de pensamiento y el estilo cognitivo dependencia-independencia de campo en estudiantes universitarios. Psicología desde el Caribe (5), 176-196. 
Ivañez, J. (2000). La gestión del diseño en la empresa. Madrid, España: McGraw Hill.

Jonassen, D. H. (2000). Toward a design theory of problem solving. Educational technology Research and Development , $48(4), 63-85$.

Jonassen, D., \& Hernandez Serrano, J. (2002). Case-based reasoning and instructional design: using stories to support problem solving. Educational Technology, Research and Development, 50 (2), 65-77.

Kim, M. H., Kim, Y. S., Lee, H. S., \& Park, J. A. (2007). An underlying cognitive aspect of design creativity: limited commitment mode control strategy. Design Studies, 28 (6), 585-604.

Kolfschoten, G., Lukosch, S., Verbraeck, A., Valentin, E., \& Vreede, G.-J. (2010). Cognitive learning efficiency through the use of design patterns in teaching. Computers \& Education (54), 652-660.

Krippendorff, K. (2006). The Semantic Turn. New York, U.S.A.: Taylor\& Francis Group.

Layton, D. (1993). Technology's challenge to science education. Buckingham, UK: Open University Press.

Liikkanen, L. A., \& Perttula, M. (2008). Exploring problem decomposition in conceptual design among novice designers. (E. Ltd., Ed.) Design studies, 30 (1), 38-59.

Maldonado, L. F., \& Quintero, V. (2006). La autorregulación como mecanismo de evaluación en el área de tecnología e

informática. En I. Instituto para la investigación y el desarrollo Pedagógico, Ambientes de aprendizaje y evaluación interlocutiva (pág. 290). Bogotá, Colombia: IDEP.

Maldonado, L., \& Andrade, E. (2001). Ambiente computarizado para el aprendizaje autodirigido del diseño ACA2 (1 ed.). Bogotá,

Colombia: Universidad Pedagógica Nacional-COLCIENCIAS.

Martínez, E. (2006). Apuntes para una pedagogía del diseño. Imaginarios, 1 (1), 12-15.

Mazzeo, C., \& Romano, A. M. (2007). La enseñanza de las disciplinas proyectuales. Buenos Aires, Argentina: Nobuko.

Meneely, J., \& Portillo, M. (2005). The Adaptable Mind in Design: Relating Personality, Cognitive Style, and Creative

Performance. Creativity Research Journal, 17 (2-3), 155-166.

Novak, J. (1982). Teoría y práctica de la educación. Alianza.

Osorio, J. C. (2008). Introducción al pensamiento sistémico. Cali: Programa editorial Universidad del Valle.

Pantoja O., M. A. (13 de diciembre de 2005). Estilos cognitivos. Creando , 13.

Parra, J. (1996). Inspiración. Bogotá, Colombia: Magisterio.

Perkins, D. (1989). Conocimiento como diseño. (F. Quebbermann, Trad.) Bogotá, Colombia: Universidad Javeriana.

Piaget, J. (1981). La toma de conciencia. Madrid, España: Morata S.A.

Piaget, J. (1994). Seis estudios de psicología. Bogotá: Drake.

Schraagen, J. M. (1993). How experts solve a novel problem in experimental design. Cognitive Science, 17 (2), $285-309$.

Spotts, J., \& Mackler, B. (1976). Relationships of field-dependent and field independent cognitive styles to creative test performance. Perceptual and Motor Skills, 24 (1), 239-268.

Sternberg, R., \& Zhang, L.-f. (2001). Perspectives on Thinking, Learning, and Cognitive Styles. New Jersey, United States of America: Lawrence Erlbaum Associates, Inc.

Stoyanov, S., \& Kirschner, P. (2007). Effect of problem solving support and cognitive styles on idea generation: implications for technology-enhanced learning. Journal of Research on Technology in Education, 1 (40), 49-63.

Treffinger, D., Selby, E. C., \& Isaksen, S. G. (23 de 11 de 2008). Understanding individual problem-solving style: a key to learning and applying creative problem solving. Learning and Individual Differences , 390-401.

Turner, S. (2009). ASIT- a problem solving strategy for education and eco-friendly sustainable design. International Journal of

Technology Design Education (19), 221-235.

Vargas G., G. (1999). Filosofía, Pedagogía, Tenología. Bogotá: U. San Buenaventura.

Vigotsky, L. (1995). Pensamiento y lenguaje. Buenos Aires, Argentina: Fausto.

Vigotsky, L. (1996). El desarrollo de los procesos psicológicos superiores (1 ed.). Barcelona, España: Crítica.

Wiener, N. (1995). Inventar. Barcelona, España: Tusquets.

Witkin, H. A., Moore, C. A., Goodenough, D. R., \& Cox, P. W. ( 1977). Field-Dependent and Field-Independent Cognitive Styles and Their Educational Implications. Review of Educational Research, 47 (1), 1-64.

Witkin, H., \& Goodenough, D. (1985). estilos cognitivos, naturaleza y origenes. Madrid: Piramide.

Recibido: septiembre 30 / Aprobado: diciembre 1 de 2012 